\title{
FINITE-TIME BLOW-UP OF THE HEAT FLOW OF HARMONIC MAPS FROM SURFACES
}

\author{
KUNG-CHING CHANG, WEI-YUE DING \& RUGANG YE
}

This note is concerned with the singular behaviour of solutions to the heat equation of harmonic maps between Riemannian manifolds. Eells and Sampson [5] showed that for $C^{1}$ initial values the solutions exist locally in time and asked whether they exist for all time or they may blow up in finite time. Recently, Coron-Ghidaglia [3], Ding [4] and Chen-Ding [2] constructed many examples of finite-time blow-up of the solutions in the case where the domain manifold has dimension greater than two.

However, it has been widely believed that finite-time singularities would not occur in dimension two. Such a belief is seemingly supported by a number of phenomena special for dimension two. For example, it has been noted that even if a given smooth map from a surface belongs to a homotopy class containing no harmonic maps, the solution to the heat equation with the given map as initial value may exist globally (see [1] and [6]). Such a phenomenon is unknown in higher dimensions and may be viewed as an indication that probably all solutions exist globally in dimension two.

Another phenomenon, observed first by R. Hamilton, is as follows. Suppose that for some point $x_{0}$ of the domain surface and $t_{0}>0$ one has

$$
\bar{e}(t) \equiv\|e(u(t, \cdot))\|_{C^{0}\left(D_{\delta}\right)} \leq \frac{C}{t_{0}-t}, \quad \text { for } t<t_{0},
$$

where $u$ is a solution of the heat equation, $e(u(t, \cdot))$ denotes the energy density of $u$ at $t$, and $D_{\delta}$ is a small neighborhood of $x_{0}$. Then, using rescaling analysis (cf. [8]) it is not hard to show that $\left(t_{0}, x_{0}\right)$ is a regular point of the solution $u$. Roughly speaking, this implies that if a solution blows up at some finite time $t_{0}$, then the velocity of the blow-up cannot be very high. Indeed, by letting $\lambda(t)=1 / \bar{e}(t)$, the above inequality is equivalent to

$$
\lambda(t) \geq C^{-1}\left(t_{0}-t\right),
$$

Received August 6, 1991. The first and second authors were partially supported by the Chinese National Science Foundation, and the third author by the National Science Foundation. 
and blow-up occurs at $t_{0}$ iff $\lambda(t) \rightarrow 0$ as $t \rightarrow t_{0}$. The above observation says that $\lambda(t)$ cannot go to 0 as fast as any linear function. This phenomenon seems to be rather unique compared with several other geometric heat equations such as the Ricci flow and the mean curvature flow.

Finally, philosophically one expects that the two-dimensional heat flow of harmonic maps should have special behavior due to the conformal invariance of the harmonic map problem in dimension two. Helein [7] proved very recently that any weakly harmonic maps from surfaces are smooth. This also gives one some hope for establishing a similar regularity result for the global weak solutions [8] to the heat equation of harmonic maps from surfaces.

Nevertheless, it turns out that finite-time blow-ups do occur in dimension two, as will be shown by simple examples in the following. The examples of finite-time blow-up which we will construct are for solutions to the heat equation of harmonic maps from the unit disk $D^{2}$ into the unit 2-sphere $S^{2}$. We will only consider solutions which are $S^{1}$-equivariant in space variables, just as in [1]. We will also show that our proof can be used to produce similar examples for maps from $S^{2}$ onto $S^{2}$ of degree $\geq 3$ (or $\leq-3)$.

Let

$$
D^{2}=\left\{x=\left(x_{1}, x_{2}, 0\right) \in R^{3}:|x|^{2} \leq 1\right\}, S^{2}=\left\{x \in R^{3}:|x|^{2}=1\right\} .
$$

Given $u_{0} \in C^{1}\left(D^{2}, S^{2}\right)$, we consider the initial-boundary value problem for the heat equation of harmonic maps as follows.

$$
\left\{\begin{array}{l}
\frac{\partial u}{\partial t}=\Delta u+|\nabla u|^{2} u, \\
u(0, x)=u_{0}(x), \\
\left.u(t, \cdot)\right|_{\partial D^{2}}=\left.u_{0}\right|_{\partial D^{2}} .
\end{array}\right.
$$

It is well known that (1) admits a unique classical solution $u$ that solves the problem on $[0, T) \times D^{2}$, where $T=T\left(u_{0}\right) \in(0, \infty]$ is the maximal existence time of $u$. If $T<\infty$, we say that the solution $u$ blows up in finite time. In [1], it is shown that if the initial map has the following symmetric form

$$
u_{0}(x)=\left(\frac{x}{|x|} \sin h_{0}(|x|), \cos h_{0}(|x|)\right),
$$

where $h_{0} \in C^{1}([0,1])$ and $h_{0}(0)=0$, then the solution $u$ of $(1)$ has the form

$$
u(t, x)=\left(\frac{x}{|x|} \sin h(t,|x|), \cos h(t,|x|)\right),
$$


and the problem (1) reduces to the following problem for $h(t, r)$ :

$$
\left\{\begin{array}{l}
\frac{\partial h}{\partial t}=h_{r r}+\frac{1}{r} h_{r}-\frac{\sin h \cos h}{r^{2}}, \\
h(0, r)=h_{0}(r), \\
h(t, 0)=h_{0}(0)=0, \quad h(t, 1)=h_{0}(1)=b .
\end{array}\right.
$$

In [1], the authors show that if $\left|h_{0}\right| \leq \pi$, then the solution $h$ of (3) exists for all $t>0$; consequently the solution $u$ of (1) with initial map given by (2) is a global solution. (See also [6].) In contrast to this result, we prove here the following theorem.

Theorem. Suppose that $u_{0} \in C^{1}\left(D^{2}, S^{2}\right)$ has the form (2) with $h_{0}(0)=$ 0 and $\left|h_{0}(1)\right|>\pi$. Then the solution $u$ of (1) with the given initial map $u_{0}$ blows up in finite time.

Proof. We may assume that $h_{0}(1)=b>\pi$, for otherwise $-h$ is a solution of the same equation satisfying the assumption. It is easy to show that if $T_{0}$ is the maximal existence time for the solution $u$ of (1), then it is the maximal time such that

$$
h \in C^{0}\left(\left[0, T_{0}\right) \times[0,1]\right) \cap C^{\infty}\left(\left(0, T_{0}\right) \times[0,1]\right) .
$$

We need only to prove that such a solution $h$ of (2) must blow up in finite time, i.e. $T_{0}<\infty$. We will treat some special cases before proving the theorem in its full generality.

(i) We first prove the theorem for a class of initial data $h_{0}$ that satisfy a certain condition to be specified below (see (13)). The proof in this case is simple and for those who wish to see just one example of finitetime blow-up it will suffice. To this end we need to construct a family of subsolutions to the parabolic equation in (3) that blow up in finite time. For each subsolution $f$ in this family there exists some $T=T(f)>0$ such that $f \in C^{2}((0, T) \times(0,1]) \cap C^{1}([0, T) \times[0,1])$ and satisfies

$$
\frac{\partial f}{\partial t} \leq f_{r r}+\frac{1}{r} f_{r}-\frac{\sin f \cos f}{r^{2}}, \quad \text { on }[0, T) \times(0,1) \text {. }
$$

We will denote the right-hand side of $(4)$ by $\tau(f)$ for simplicity, and consider the ordinary differential equation

$$
\tau(\phi) \equiv \phi_{r r}+\frac{1}{r} \phi_{r}-\frac{\sin \phi \cos \phi}{r^{2}}=0,
$$

which can be solved explicitly. Indeed, all the solutions of (5) satisfying $\phi(0)=0$ and $\phi(r)>0$ for $r>0$ are given by

$$
\phi(r, \lambda)=\arccos \left(\frac{\lambda^{2}-r^{2}}{\lambda^{2}+r^{2}}\right), \quad \lambda>0 \text {. }
$$


Notice that for $r>0$

$$
\lim _{\lambda \rightarrow 0} \phi(r, \lambda)=\pi, \quad \text { and } \quad \lim _{\lambda \rightarrow \infty} \phi(r, \lambda)=0 .
$$

Let $a=1+\varepsilon$, where $\varepsilon \in(0,1)$. We introduce a family of new functions

$$
\theta(r, \lambda) \equiv \phi\left(r^{a}, \lambda\right)=\arccos \left(\frac{\lambda^{2}-r^{2 a}}{\lambda^{2}+r^{2 a}}\right),
$$

and observe that they are solutions to the equation

$$
\theta_{r r}+\frac{1}{r} \theta_{r}-\frac{a^{2} \sin \theta \cos \theta}{r^{2}}=0 .
$$

Set

$$
\theta(r)=\theta(r, \mu)
$$

where $\mu>0$ will be chosen appropriately large so that $\theta$ can be as small as we want. For the present, we assume $\theta(r)$ to satisfy

$$
\cos \theta(r) \geq \frac{1}{1+\varepsilon} \quad \text { for } r \in[0,1]
$$

Next, let $\lambda(t)$ be the function defined by the following ordinary differential equation:

$$
\lambda^{\prime}=-\delta \lambda^{\varepsilon}, \quad \lambda(0)=\lambda_{0},
$$

where $\delta, \lambda_{0}>0$ are numbers to be determined. The subsolution $f$ has the form

$$
f(t, r)=\phi(r, \lambda(t))+\theta(r)
$$

Solving (9) we get

$$
\lambda(t)=\left[\lambda_{0}^{1-\varepsilon}-(1-\varepsilon) \delta t\right]^{1 /(1-\varepsilon)} .
$$

Set

$$
T=\lambda_{0}^{1-\varepsilon} /(1-\varepsilon) \delta .
$$

Then $\lambda(t)$ is smooth on $[0, T)$, and $\lambda(t) \rightarrow 0$ as $t \rightarrow T$. We will call $T$ the blow-up time of $f$ since

$$
\begin{gathered}
f_{r}(t, r)=\phi_{r}(r, \lambda(t))+\theta_{r}(r)=\frac{2 \lambda(t)}{\lambda^{2}(t)+r^{2}}+\frac{2 a \mu r^{2 \varepsilon}}{\mu^{2}+r^{2 a}}, \\
f_{r}(t, 0)=\frac{2}{\lambda(t)},
\end{gathered}
$$

and hence

$$
\lim _{t \rightarrow T} f_{r}(t, 0)=+\infty
$$


We claim that there exists $\delta_{0}=\delta_{0}(\varepsilon, \mu)>0$ such that the function $f$ satisfies (4) provided $\delta \leq \delta_{0}$. Indeed, since $f$ is of the form $f=\phi+\theta$ and $\phi, \theta$ satisfy equations (5), (7) respectively, we have

$$
\begin{aligned}
\tau(f) & =r^{-2}\left[\sin \phi \cos \phi-\sin (\phi+\theta) \cos (\phi+\theta)+(1+\varepsilon)^{2} \sin \theta \cos \theta\right] \\
& =r^{-2}\left[(1+\varepsilon)^{2} \sin \theta \cos \theta-\cos (2 \phi+\theta) \sin \theta\right] \\
& \geq r^{-2}[(1+\varepsilon) \sin \theta-\cos (2 \phi+\theta) \sin \theta] \\
& \geq \frac{\varepsilon \sin \theta}{r^{2}},
\end{aligned}
$$

where we have used (8). An easy computation yields that

$$
\sin \theta=\frac{2 \mu r^{a}}{\mu^{2}+r^{2 a}} \geq \frac{2 \mu r^{1+\varepsilon}}{\mu^{2}+1}
$$

Thus,

$$
\tau(f) \geq \varepsilon_{1} r^{\varepsilon-1},
$$

where $\varepsilon_{1}=2 \mu \varepsilon /\left(\mu^{2}+1\right)$. On the other hand, we have

$$
\frac{\partial f}{\partial t}=\frac{\partial}{\partial t} \phi(r, \lambda(t))=\lambda^{\prime}(t) \frac{\partial \phi}{\partial \lambda}(r, \lambda(t))=-\delta \lambda^{\varepsilon} \cdot \frac{-2 r}{\lambda^{2}+r^{2}}=\frac{2 \delta \lambda^{\varepsilon} r}{\lambda^{2}+r^{2}} .
$$

From the above two inequalities we see that (4) is true if

$$
\frac{2 \delta \lambda^{\varepsilon} r}{\lambda^{2}+r^{2}} \leq \varepsilon_{1} r^{\varepsilon-1}, \quad \forall \lambda>0 \text { and } r \in(0,1) .
$$

Introducing the new variable $s=r / \lambda \in(0, \infty)$, it is easy to see that (11) is equivalent to

$$
g(s) \equiv \frac{s^{2-\varepsilon}}{1+s^{2}} \leq \varepsilon_{1} / 2 \delta, \quad \forall s>0 .
$$

It is clear that the function $g(s)$ has a maximum $M(\varepsilon)$ depending only on $\varepsilon$. Thus if $\delta \leq \varepsilon_{1} / 2 M(\varepsilon)$, then the above inequality holds. This proves our claim. Moreover, we may require the subsolution $f$ to satisfy

$$
f(t, 1) \leq b \text { for } t \in[0, T) .
$$

In fact, since $0<\phi<\pi,(12)$ holds provided that we choose the $\theta$ small so that $\theta(1) \leq b-\pi$.

Now we assume that for some $\bar{t} \geq 0$ the solution $h$ of (3) satisfies

$$
h(\bar{t}, r) \geq f(0, r) \text { for } r \in[0,1] .
$$


In view of (4), (12), $f(t, 0)=0$ and (13), $f$ is a subsolution for the solution $\bar{h}(t, r) \equiv h(\bar{t}+t, r)$ of problem (3). If $T_{0}>\bar{t}+T$, then by the maximum principle (cf. $\S 2$ of [1]) we have

$$
h(\bar{t}+t, r)>f(t, r) \text { for } r \in(0,1), t \in(0, T) .
$$

Since $h(\bar{t}+t, 0)=f(t, 0)=0$, this implies

$$
h_{r}(\bar{t}+t, 0) \geq f_{r}(t \cdot 0) \quad \text { for } t \in(0,1) .
$$

In view of $(10)$, we see that $h_{r}(t, 0)$ cannot be bounded up to time $\bar{t}+T$. It follows that we must have $T_{0} \leq \bar{t}+T$, i.e. $h$ blows up in finite time. We remark that if $h_{0}(r) \geq f(0, r)$ (such initial values certainly exist), then (13) holds with $\bar{t}=0$, and we have proved the theorem in this case.

(ii) Next, we assume that for some $t_{0} \geq 0$

$$
h\left(t_{0}, r\right) \geq 0, \quad r \in[0,1] .
$$

We will show that if the maximal existence time $T_{0}=\infty$, then there exist $\bar{t}>t_{0}$ and a subsolution $f$ in the family of subsolutions constructed in case (i) such that (13) holds. Thus by the result of case (i) we see that $T_{0}=\infty$ cannot be true.

We first note that $h$ must blow up at infinity in the sense that

$$
\limsup _{t \rightarrow \infty}\left\|h_{r}(t, \cdot)\right\|_{C^{0}([0,1])}=\infty
$$

For otherwise, there will be a sequence $t_{k} \rightarrow \infty$ such that $u\left(t_{k}, \cdot\right)$ converges to a harmonic map as $k \rightarrow \infty$ (cf. [1]). It follows that $h\left(t_{k}, \cdot\right)$ converges to a solution $\phi$ of (5) satisfying

$$
\phi(0)=0, \quad \phi(1)=b .
$$

As we have noted, all such solutions are given by $\phi=\phi(\cdot, \lambda)$ in $(6)$. It is clear that $\phi(1, \lambda)<\pi$ for any $\lambda>0$. Hence the boundary condition (15) cannot be satisfied. This shows that $h$ must blow up. Then we deduce from Lemma 3.4 in [1] that for some sequences $t_{k} \rightarrow \infty$ and $\lambda_{k} \rightarrow 0$

$$
h\left(t_{k}, \lambda_{k} r\right) \rightarrow \beta(r) \text { in } C^{2}([0, R])
$$

for any $R>0$, where $\beta(r)= \pm \phi(r, 1)$. But (14) implies that

$$
h(t, r)>0 \text { for } t>t_{0} \text { and } r \in(0,1]
$$

by the maximum principle (see Lemma 2.3 in [1]). So we have $\beta(r)=$ $\phi(r, 1)$. Since $\phi_{r}(0,1)=2$, it follows from (16) that we can choose $\bar{t}=t_{k}$ for some large $k$ so that $\bar{t}>t_{0}$ and

$$
h_{r}(\bar{t}, 0) \geq 1 \text {. }
$$


It is not hard to see from (17), (18) and the construction of our subsolutions that we may choose the parameters $\lambda_{0}$ and $\mu$ so large that the subsolution $f$ satisfies (13). As noted above, this implies the solution $h$ blows up in finite time.

(iii) Finally we show that if $T_{0}=\infty$, then we can find some $t_{0} \geq 0$ such that (14) holds. In view of the result of case (ii), this will imply that the solution $h$ blows up in finite time.

First, we choose a subsolution

$$
f_{1}(t, r)=\phi\left(r, \lambda_{1}(t)\right)+\theta_{1}(r)
$$

with blow-up time $T_{1}$ in the subsolution family constructed in (i). Let $n$ be a positive integer such that

$$
h_{0}(r) \geq f_{1}(0, r)-n \pi \quad \text { for } r \in[0,1] .
$$

Then one checks easily that $F_{1}(t, r) \equiv f_{1}(t, r)-n \pi$ satisfies the differential inequality (4). Moreover, it satisfies

$$
F_{1}(0, r) \leq h_{0}(r), \quad F_{1}(t, 0)=-n \pi<0, F_{1}(t, 1)<b-n \pi<b .
$$

By the maximum principle, we have

$$
h(t, r) \geq F_{1}(t, r)=\phi\left(r \lambda_{1}(t)\right)+\theta_{1}(r)-n \pi, \quad r \in[0,1], t \in\left[0, T_{1}\right) .
$$

Since $\phi\left(r, \lambda_{1}(t)\right) \rightarrow \pi$ as $t \rightarrow T_{1}$ for $r>0$,

$$
h\left(T_{1}, r\right) \geq \theta_{1}(r)-(n-1) \pi, \quad r \in[0,1] .
$$

If $n=1$, then (19) implies (14) with $\bar{t}=T_{1}$, and the proof has been finished. If $n>1$ we see that since $h\left(T_{1}, 0\right)=0>-(n-1) \pi,(19)$ yields

$$
h\left(T_{1}, r\right)>-(n-1) \pi \text { for } r \in[0,1] \text {. }
$$

By the continuity of $h\left(T_{1}, \cdot\right)$ there exists $\alpha_{1}>0$ such that

$$
h\left(T_{1}, r\right) \geq \alpha_{1}-(n-1) \pi \quad \text { for } r \in[0,1] .
$$

Thus we may choose a subsolution $f_{2}(t, r)$ with sufficiently large parameters $\lambda_{0}$ and $\mu$ such that

$$
f_{2}(0, r) \leq \alpha_{1} \text { for } r \in[0,1] .
$$

Let $T_{2}$ be the blow-up time of $f_{2}$. Then one checks that $F_{2}(t, r) \equiv$ $f_{2}(t, r)-(n-1) \pi$ is a subsolution to the equation in (3) with

$$
F_{2}(0, r) \leq h\left(T_{1}, r\right), \quad F_{2}(t, 0)=-(n-1) \pi<0, \quad F_{2}(t, 1)<b,
$$

and proves as above that

$$
h\left(T_{1}+T-2, r\right) \geq \theta_{2}(r)-(n-2) \pi, \quad r \in[0,1] .
$$


If $n=2$ we are done. If $n>2$ we continue this procedure until (14) is satisfied for some $\bar{t}=T_{1}+\cdots+T_{n}$. This completes our proof of the theorem.

Finally we would like to remark on how to construct similar examples for the case of $S^{2} \rightarrow S^{2}$. We first note that if $S_{+}^{2}$ is the upper closed hemisphere of $S^{2}$, then $S_{+}^{2}$ is isometric to $\left(D^{2}, \rho(|x|) d s^{2}\right)$, where $d s^{2}$ denotes the Euclidean metric of $R^{2}$ and

$$
\rho(|x|)=\frac{4}{\left(1+|x|^{2}\right)^{2}} .
$$

Thus, when we consider the heat equation of harmonic maps from $S_{+}^{2}$ into $S^{2}$, we may choose coordinates on $S_{+}^{2}$ so that the equation becomes

$$
\left\{\begin{array}{l}
\frac{\partial u}{\partial t}=\rho(|x|)^{-1}\left(\Delta u+|\nabla u|^{2} u\right) \\
u(0, x)=u_{0}(x) \\
u(t, \cdot)\left|\partial D^{2}=u_{0}\right| \partial D^{2}
\end{array}\right.
$$

Similarly, when $u_{0}$ takes the form (2), the problem is reduced to

$$
\left\{\begin{array}{l}
\frac{\partial h}{\partial t}=\rho(r)^{-1}\left(h_{r r}+\frac{1}{r} h_{r}-\frac{\sin h \cos h}{r^{2}}\right) \\
h(0, r)=h_{0}(r), \\
h(t, 0)=0, \quad h(t, 1)=b .
\end{array}\right.
$$

Since $1 \leq \rho(r) \leq 4$ for $r \in[0,1]$, the proof of the theorem can be applied to show that if $|b|>\pi$ then the solution $h$ of (21) must blow up in finite time. This also gives examples of finite-time blow-up for (20). Now, if (i) $b=k \pi$ or (ii) $b=(k-(1 / 2)) \pi$ for $k=2,3, \cdots$, then the map $u_{0}$ considered as a map from $S_{+}^{2}$ satisfies (i) $u_{0}\left(\partial S_{+}^{2}\right)=$ the north pole or the south pole, or (ii) $u_{0}\left|\partial S_{+}^{2}=i d\right| \partial S_{+}^{2}$, respectively. In these cases $u_{0}$ can be extended to a map from $S^{2}$ as follows. Let $R_{1}, R_{2}: S^{2} \rightarrow S^{2}$ be defined by

$$
R_{1}\left(x_{1}, x_{2}, x_{3}\right)=\left(x_{1}, x_{2},-x_{3}\right), \quad R_{2}\left(x_{1}, x_{2}, x_{3}\right)=\left(-x_{1},-x_{2},-x_{3}\right) .
$$

Then the definition of $u_{0}$ can be extended to the lower hemisphere by requiring

$$
u_{0} \circ R_{1}=R_{2} \circ u_{0} \quad \text { in case }(\mathrm{i})
$$

and

$$
u_{0} \circ R_{1}=R_{1} \circ u_{0} \text { in case (ii). }
$$


The resulting map denoted by $\tilde{u}_{0}$ will be in $C^{1}\left(S^{2}, S^{2}\right)$, and has degree $2 k$ in case (i), degree $2 k-1$ in case (ii). Similarly, for the solution $u(t, x)$ of (20) with initial value $u_{0}$, we can extend its domain of space variables to the whole $S^{2}$ in the same way. Denote the resulting mapping by $\tilde{u}$. Then, noting that $R_{1}, R_{2}$ are isometries of $S^{2}$, one checks in a standard way that $\tilde{u}$ is a solution of the heat equation for harmonic maps from $S^{2}$ into $S^{2}$ with initial value $\tilde{u}_{0}$. Since the solution $u$ of (20) blows up in finite time and $\tilde{u}(t, x)$ coincides with $u(t, x)$ for $x \in S_{+}^{2}$ by construction, we see that $\tilde{u}$ blows up in finite time. This completes our sketch for the examples of finite-time blow-up in the case of $S^{2} \rightarrow S^{2}$.

Acknowledgment. The third author is grateful to the Institute of Mathematics, Peking University, for its hospitality.

\title{
References
}

[1] K.-C. Chang \& W.-Y. Ding, A result on the global existence for heat flows of harmonic maps from $D^{2}$ into $S^{2}$, Nemantics, J.-M. Coron et al. ed., Kluwer Academic Publishers, 1990, 37-48.

[2] Y. Chen \& W.-Y. Ding, Blow-up and global existence for heat flows of harmonic maps, Invent. Math. 99 (1990) 567-578.

[3] J.-M. Coron \& J.-M. Ghidaglia, Explosion en temps fini pour le flot des applications harmoniques, C. R. Acad. Sci. Paris Ser. I 308 (1989) 339-344.

[4] W.-Y. Ding, Blow-up of solutions of heat flows for harmonic maps, Advances in Math. 19 (1990) 80-92.

[5] J. Eells \& J. H. Sampson, Harmonic mappings of Riemannian manifolds, Amer. J. Math. 86 (1964) 109-169.

[6] M. Grayson \& R. Hamilton, preprint, 1989.

[7] F. Helein, Regularite des applications faiblement harmoniques entre une surface et une variete Riemannienne, preprint, 1991.

[8] M. Struwe, On the evolution of harmonic maps of Riemannian surfaces, Math. Helv. 60 (1985) 558-581.

\author{
Peking University \\ ACADEMIa Sinica \\ University of California at SANTA Barbara
}


